\title{
Study of Efficacy of Titanium Three- Dimensional Locking Plate In The Treatment of Mandibular Fractures
}

\author{
Pavan Kumar $\mathrm{B}^{1}$, Yashwanth Yadav², Venkatesh V³, Brahmaji Rao J ${ }^{4}$
}

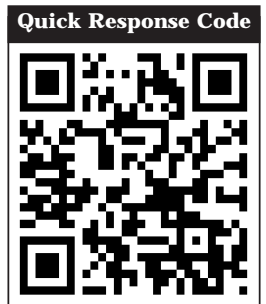

doi: $10.5866 / 2016.8 .10222$

${ }^{1}$ Professor and HOD

2Senior Lecturer

3\&4Professor

Department of Oral and Maxillofacial Surgery,

Kamineni Institute of Dental Sciences, Narketpally.

\section{Article Info:}

Received: October 8, 2016

Review Completed: November 10, 2016

Accepted: December 11, 2016

Available Online: December, 2016 (www.nacd.in)

(C) NAD, 2016 - All rights reserved

\section{Email for correspondence:}

pavankumarbatchu40@gmail.com

\begin{abstract}
:
Context: Face is the window through which we perceive the world around us and the world perceive us. In the era of increasing automobilization and industrialization the treatment of mandibular fractures has attained a prominent position. The main goal in the treatment of fracture is to restore pre injury anatomical form, with associated aesthetics and function.
\end{abstract}

Aim: The purpose of the study is to evaluate the efficacy of Titanium Three Dimensional Locking Plates in the treatment of mandibular fractures.

Methods and Materials: Total twenty patients wereselected who suffered with mandibular fractures randomly. All patients were operated through the standard intra oral incision at fracture site of the mandible and immobilized the fracture segments using titanium three dimensional locking plating systems. The resulting osteosynthesis were evaluated with the scoring system for 9 parameters and complications encountered during their use were also recorded and reported in this study

Results: The results of this study suggest that fixation of mandibular fracture with 3D plates provides three-dimensional stability and carries low morbidity and infection rates. These plates functions as internal fixator achieving stability by locking thescrew to the plate, the other advantages offered by them are less screw loosening, less precision in plate adaptation and less alteration of the osseous or ocdusal relationship upon screw tightening.

Conclusion: During the course of present study, 3D plates stabilize the bone fragments in three dimensions because of cl osed quadrangular geometric shape and the ease of contouring and adapting. The 3D plate was found to be standard in profile, strong yet malleable, facilitating reduction and stabilization at both the superior and inferior borders giving three-dimensional stability at fracture site. But the small sample size and limited follow up could be considered as the limitations of our study. It is hence recommended to have a multicenter study with large number of patients and correlation among these studies to authenticate our claims.

Key words: Titanium Three Dimensional Locking Plate System, Mandibular Fractures, Open Reduction Internal Fixation (ORIF). 


\section{Introduction:}

Fractures of the facial skeleton can be studied and described in anatomic terms, by functional considerations, by treatment strategies and by outcome measures. Functional and morphologic characteristics that are unique to lower facial skeleton must beconsidered. Theaim of mandibular fracture treatment is the restoration of anatomical form and function, with particular care to establish the occlusion. ${ }^{1}$ The present era of fast moving, result oriented life has made a definite impact on the common man. Individuals have begun to work harder, faster and become more aggressive towards reaching their goals. Maxillofacial trauma is very common in all these unforeseen events and the unique position of the mandible on the face makes it vulnerable. It is therefore, one of the most commonly fractured facial bones. ${ }^{2}$ Methods of open reduction and internal fixation have changed and diversified enormously in the past few years. ${ }^{3}$ With the development of osteosynthesis in maxillofacial surgery different systems have been designed. They have become smaller, simpler to handle and extra oral incisions can be avoided. Miniplate fixation of mandibular fractures has become a standard. Even though the idea of plate osteosynthesis is more than 100 years old, more research is needed for optimal treatment. ${ }^{4}$ When selecting a fixation scheme for a fracture one has to consider many things such as the size, number of fixation devices, their location, ease of adaptation and fixation, biomechanical stability, the surgical approach, the amount of soft tissue disruption necessary to expose the fracture and place the fixation devices. Research continues to focus on the size, shape, number, and biomechanics of plate/screw systems to improve surgical outcomes. ${ }^{3,5}$ With the above considerations, in recent times two systems have been studied commonly namely 3 dimensional (3D) and the locking miniplate with its comparison to conventional miniplates using Champy's principle. ${ }^{6-}$ 10

The 3D plating system is based upon the principle of obtaining support through geometrically stable configuration. The quadrangle geometry of plates assures a good stability in three dimensions of the fracture site since it offers good resistance against torque forces. ${ }^{6}$

The present study was designed with an aim of evaluating the efficacy of 3D Titanium Locking Plates in the treatment of Mandibular Fracture and to report the complications encountered during its use.

\section{Materials and Methods:}

The present study consisted sample of twenty patients who reported with fractures of mandible and were treated over a period between September 2011 and August 2013. Out of twenty patients 16 were male and 4 were female patients.

Criteria for case selection were Patients with fractures of the mandible between the two mental foramina and angles of the mandible and low level subcondylar fractures, patients above the age of 17 years, patients without significant history of medical disorders, patients reporting to the department within 7 days of trauma (Figure 1). The following cases were not included in the study, patients below 17years of age, medically compromised patients, gross infection at the site of fracture, more than 7 days after injury. The radiographic evaluation consisted of OPG (Figure 2), and in required cases CT-scans with $3 \mathrm{D}$ reconstruction were taken preoperatively. While a post-operative OPG was taken on third day, fifteenth day, one month, two months, and three months. Post-operative OPG is planned to assess the approximation of the fracture fragment and to check loosening of screws and fracture of the plate.

\section{Procedure:}

An intraoral vestibular incision was given extending to about $2 \mathrm{~cm}$ on either side of the fracture line. The mucoperiosteal flap was then raised and the fracture site was exposed (Figure 3 ) and curetted with the hel $p$ of curetteto remove granulation tissue and blood clots. The fracture site was irrigated with $5 \%$ povidine iodine foll owed by normal saline. In the mandibular angle region, a mucoperiosteal incision was made over the external oblique ridge. In the 
parasymphysis region, a vestibular mucosal incision was made obliquely through the mentalis muscle, with care taken to isolate the labial branches of mental nerve. After adequate exposure the fracture site, the segments were manipulated and reduced to normal anatomic position. Dental occlusion was checked, after achieving occlusion temporary IMF was done.

\section{Adaptation and Fixation of Plate:}

Plate was adapted to the underlying bone but it does not require the kind of adaptation required in conventional plate. Care was taken to center the hole on the plate and hole made perpendicular to bone surface. The 3 Dimensional locking plates were adapted across the fracturesite (Figure 4). Thescrew hol es were made with $1.5 \mathrm{~mm}$ drill bit, perpendicular to the surface of bone under copious salineirrigation. Plates were fixed with $2 \times 10 \mathrm{~mm}$ titanium locking screws.

Following reduction, $2.0 \mathrm{~mm} 3$ Dimensional titanium locking miniplates were placed along the osteosynthesis line as described by Champy. During drilling, the adapted plate was held firmly against the bone with the plate holding forceps. The patients were evaluated pre-operatively, immediate post operatively, 15days, 1month, 2months, and 3months postoperatively. Each parameter was evaluated with the help of a scoring system on every visit of the patient. The parameters were - Operating time, Occlusion (Figure 5), Need for the post-operative IMF, Infection, Mobility of fracture segment, Pain, Wound dehiscence, Neurological deficit, Implant Failure.

\section{Grading Systems:}

1. Operating time taken: In all the cases total operating time was recorded after intubation and GA i.e. the infiltration of local anaesthesia at the site of surgery till the last suture was placed. For our study thetimetaken from adaptation of the plate to the time when the last screw placed in mandibular fracture site region was recorded.

2. Occlusion scale.

a. 1-No occlusal disturbances b. 2 -Minor occlusal disturbances

c. 3 -Severe occlusal disturbances

3. Infection scale

a. 0 - Absent

b. 1 - Present

4. Pain assessment scale

a. 0 - No pain

b. 1 -Mild pain

c. 2 -Moderate pain

d. 3-Severe pain

5. Mobility of fracture fragments (scale)

a. 0 - Absent

b. 1 - Present

6. Soft tissue dehiscence scale

a. 0 - Absent

b. 1 - Present

7. Neurological deficit scale

a. 0 - Absent

b. 1- Present

8. Implant failure

a. 0-Absent

b. 1- Present

\section{Results:}

A total of twenty patients were included in the study, out of which 16 were male and 4 were female patients. Incidence of mandibular fractures was more in males (80\%) compared to females. This may be justified by the fact that the males are generally more prone to situations in which there are high risk of trauma. In this study Parasymphysis was the most common site of fracture involving in 11 (55\%) cases, followed by Symphysis 6 (30\%), Angle 4 (20\%) and Body 3 (15\%) of mandible.

Average operating time for the adaptation and placement of each type of plate at mandibular fracture site region was noted in minutes. The total 
operating time required for the completion of either multiple or isolated surgical procedures ranged from 45 minutes to 120 minutes with a mean of 68.45 minutes and the duration of plate adaptation and fixation ranged from 4 minutes to 10 minutes with a mean of 5.95 minutes. The mean time of the operating isolated fractures was found to be 55.15 minutes, and 93.14 minutes to treat multiple fractures. The mean time taken for plate fixation was 4.46 minutes for isolated fractures and 8.71 minutes for multiple fractures. Occlusion of the patient was evaluated preoperatively and postoperatively at 1 week, 1 month, 2 months and at 3 months. Infection is the most common complication of surgical intervention. The patients were evaluated for signs of infection. The potential possibility for infection is always a consideration when treating mandibular fractures, especially when there is communication with oral cavity. Manifestations of infection include pain, mobility of fracture segment, plateloosening, sinus tract with pus discharge, wound. Preoperatively patients with gross infection at the site of fracture were excluded from the study. Postoperatively signs of infection were checked after one week, one month, two months and three months. Pain was recorded based on the visual analoguescalefor patients preoperatively and post operatively on one week, two weeks, one month, two months, three months, at mandibular fracture site. Scores for assessment of pain as follows, 0 - No pain, 1 -Mild pain, 2 - Moderate pain, 3 - Severe pain.

\section{Discussion:}

Face is the window through which we perceive the world around us and the world perceive us. In the era of increasing automobilization and industrialization the treatment of mandibular fractures has attained a prominent position. The main goal in the treatment of fracture is to restore pre injury anatomical form, with associated aesthetics and function. The goal must be accomplished by means that will produce the least disability, risk and shortest recovery period for the patient. ${ }^{11}$

The treatment of mandibular fractures has evolved over a period of time from old methods of bandaging and splinting, which are forms of closed reduction, to the more recent methods of open reduction. ${ }^{1}$ General acceptance of open osteosynthesis did not appear in maxillofacial literature, until an organized research was done by the AO group in $1950 .^{3}$ Even in open osteosynthesis technique there had been a metamorphosis and change in trends from rigid fixation in 1968, to semirigid fixation in $1973 .{ }^{12}$ Rigid fixation using dynamic compression plate had its own disadvantages such as need of very wide incision, bulky nature of plates and the procedure itself which was technique sensitive. ${ }^{13}$ Michelet (1973) ended the search for simple osteosynthesis that would guarantee fracture healing without compression. ${ }^{14}$ This was modified, developed and put to practical use by Champy in 1978. ${ }^{15} \mathrm{Fixation}$ using such plates simplified surgery and reduced surgical morbidity, however, they failed to surpass the predictability of rigid fixation using $2.4 \mathrm{~mm}$ compression and reconstruction plates. ${ }^{12}$

Farmand developed the concept of 3D miniplates. Their shape is based in the principle of quadrangle as a geometrically stable configuration for support. ${ }^{6}$ Since, the stability achieved by the geometric shape of these plates surpasses the standard miniplates. The basic form is quadrangular with $2 \times 2$ holed square plates and $3 \times 2$ (or) $2 \times 2$ rectangular plate. 3D miniplates holds the fracture segments rigidly by resisting the 3D forces namely shearing, bending and torsional forces occurring on the fracture site in function. The present study showed that the 3D plate allows no movement at the superior and inferior borders with maximal torsional and bending forces as opposed to a single linear plate applied to superior border area. Strut plates may therefore provide greater resistance against gap opening at the inferior border with biting forces compared to a single plated applied at the external oblique ridge or superior Iateral border. ${ }^{12}$

In osteosynthesis minimum of implant material with maximum stability should always be considered. The three dimensional titanium plates fulfill this requirement ideally. Due to closed 

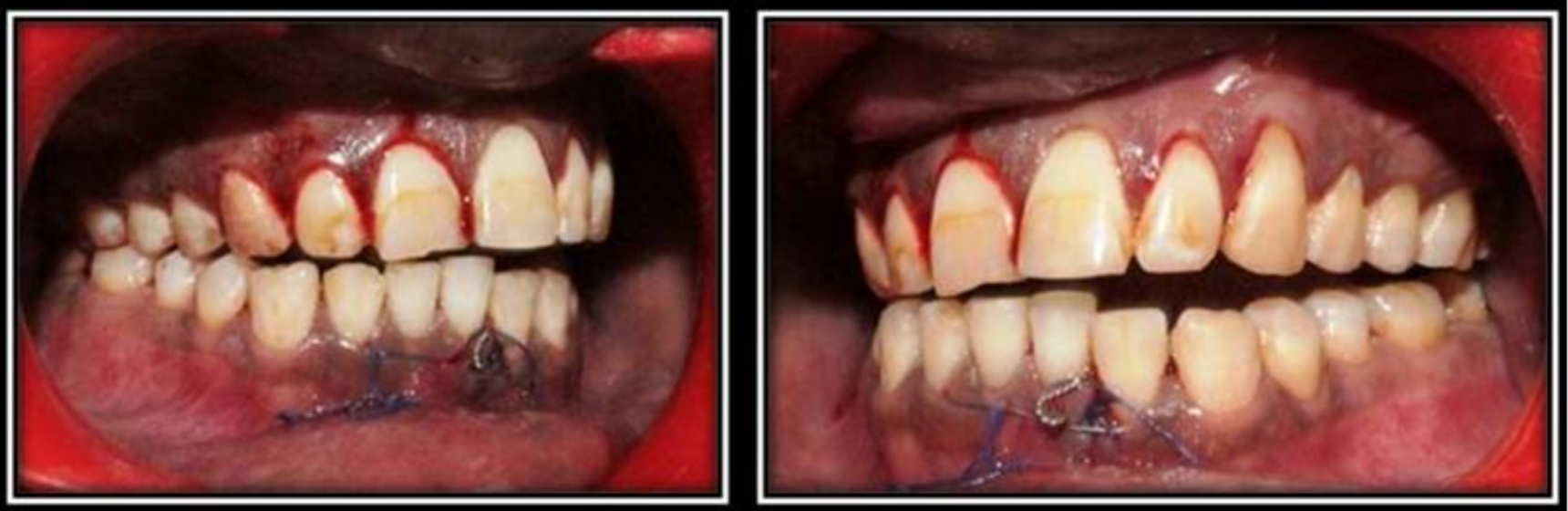

Figure 1: Right and left occlusal view showing fracture of the mandible between the two mental foramina.

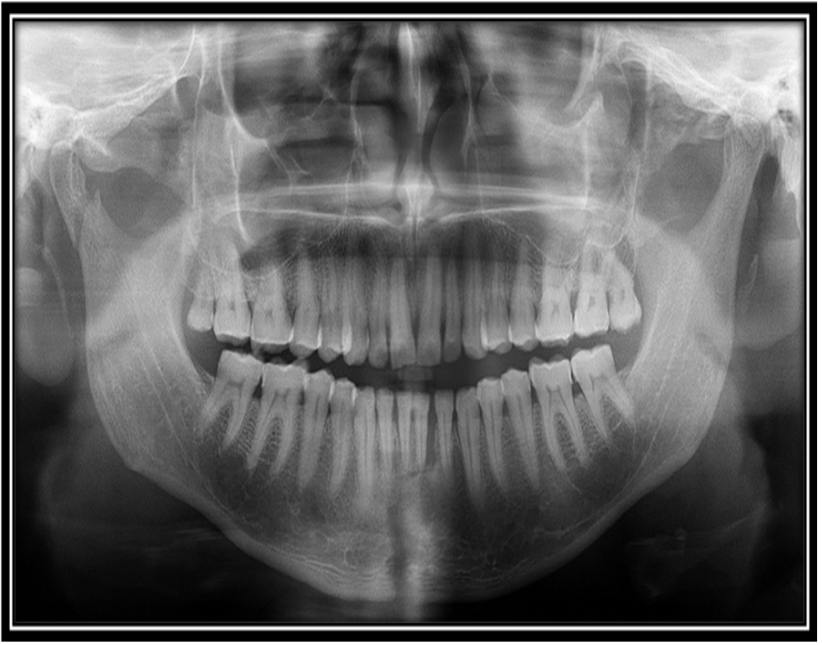

Figure 2: Pre-operative OPG of the patient showing symphysis fracture of mandible.

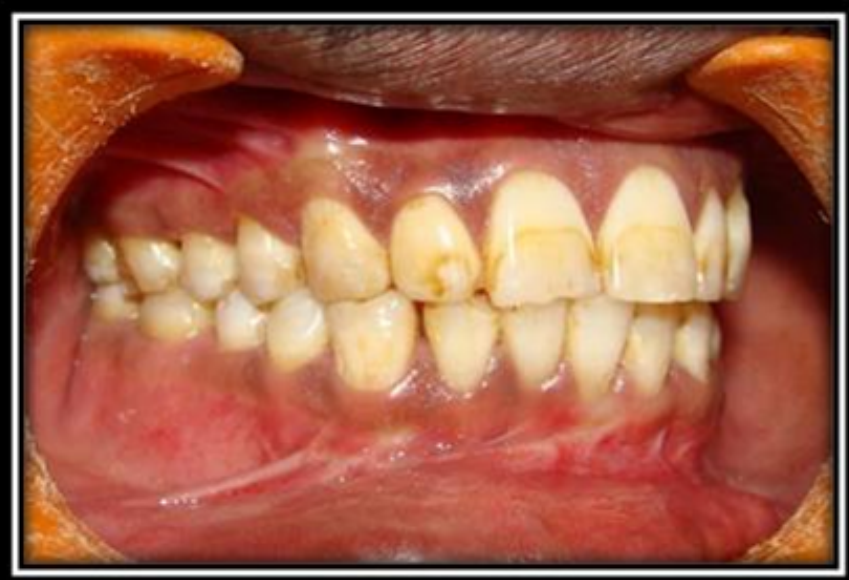

Figure 4: Placement of the $3 \mathrm{D}$ locking miniplates

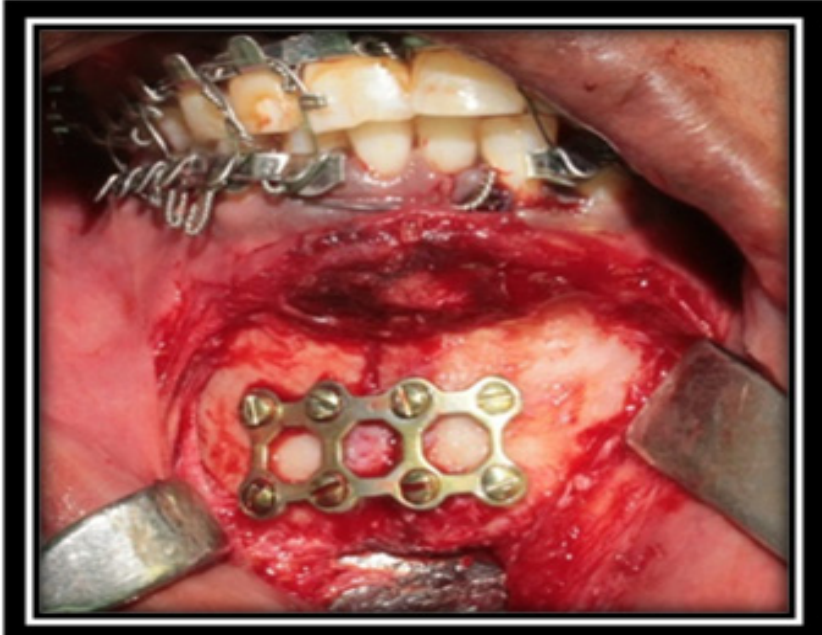

Figure 3: Incision and exposure of the fracture

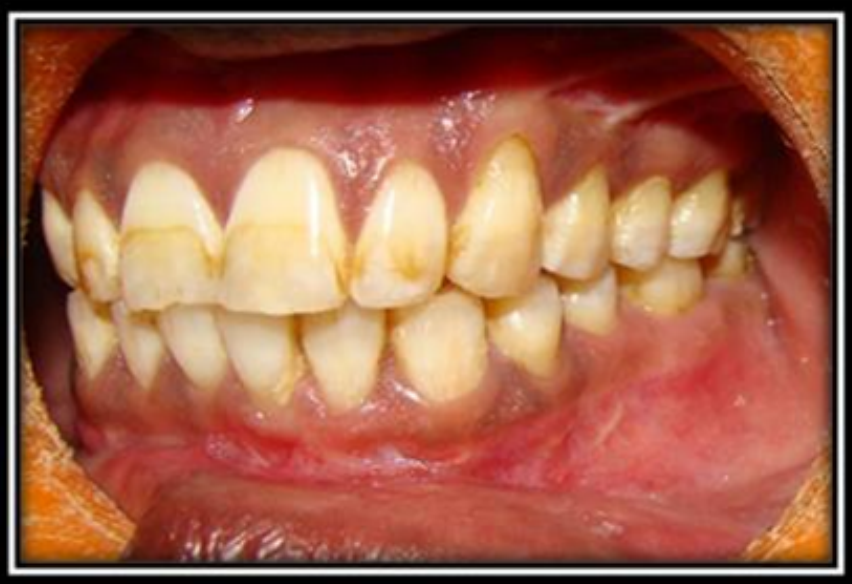

Figure 5: Right and left occlusal view showing occlusion post operatively. 
quadrangular geometric configuration of the plates, less foreign material is needed to stabilize the fragments. ${ }^{16}$

The purpose of the present study was to evaluate the efficacy of Titanium ThreeDimensional Locking Plates in the treatment of mandibular fractures. Objectives were to evaluate the efficacy of - Titanium Three Dimensional Locking Plates, to study the technique of the procedure, to evaluate and assess, occlusal stability, biomechanical stability of thefractured fragments post operatively, Ease of plate adaptation for ORIF of mandibular fractures, complications encountered, Duration of the procedure, Any need for post-operative IMF (I ntermaxillary fixation). The time required for the surgery, adaptation and fixation of the plate at the fracture site was recorded for all the patients. The total operating time required for the completion of either multiple or isolated surgical procedures ranged from 45 minutes to 120 minutes with a mean of 68.45 minutes and the duration of plate adaptation and fixation ranged from 4 minutes to 10 minutes with a mean of 5.95 minutes. The mean time of the operating isolated fractures was found to be 55.15 minutes, and 93.14 minutes to treat multiple fractures. The mean time taken for plate fixation was 4.46 minutes for isolated fractures and 8.71 minutes for multiple fractures.

FELEDY et al and ZIX et al reported a reduced average operating time of 55 minutes, single fracture site, in our study similar results are noticed (54.5minutes). ${ }^{8,17}$

3D plateis geometrically configured plate which consists of two horizontal bars interconnected with vertical bars. So single 3D plate stabilized the fracture both at superior and inferior border at a time, Hence, time is saved in plate fixation. But, in cases of oblique fracture or the fracture running through the mental foramina, more time was required for the placement of $3 \mathrm{D}$ plate. In such cases, the plate was placed either inferior or superior to the foramina, and care taken while placing the plate superior to the foramina, so that the screws are placed between the roots of the teeth. Easy application and simplified adaptation to the bone, as well as simultaneous stabilization at both the superior and inferior border makes the 3D plate a time-saving alternative to conventional miniplates.

Restoration of pre-morbid occlusion is one of the most important goals of the management of fractures in dentofacial region. The effect of not restoring the occlusion to its original condition is disabling and can cause severe effects especially on the temporo -mandibular joint.

In the present study, occlusal disturbances of the patients was assessed preoperatively and postoperatively and was graded as, minor, severe or no disturbance. There were minor occlusal disturbance in six cases, at premolar region and severe occlusal disturbance in 14 cases preoperatively. Among the six cases of minor occlusal disturbance, we achieved normal premolar and molar relationship in two patients. In four patients guiding elastics (blue) were used and the occlusal disturbance persisted even on the $15^{\text {th }}$ postoperative day, which was gradually corrected and occlusion was stabilized by the end of $1^{\text {st }}$ month postoperatively.

Out of 14 severe occlusal discrepancy cases, stable occlusion was achieved in 13 patients and only one case showed occlusal discrepancy, which persisted even during the $3^{\text {rd }}$ postoperative month.

The above results were elicited by using 3D Titanium locking plate fixation with a thorough follow up of 3 months post operatively, indicating that, 3D Titanium locking plates have a major role in achieving a stable post-operative occlusion.

Occlusal discrepancy was seen because of, imbalance between the muscular activity of the muscles of mastication, after trauma and due to edema in TMJ region. However 4 cases required postoperative MMF due to condylar fractures, which were not treated by ORIF owing to the undisplacement.

Out of twenty patients, 16 patients did not required MMF postoperatively, because we were able to achieve ideal premolar and molar occlusion. Whereas, four cases required postoperative MMF 
due to condylar fractures al ong with the Symphysis and Parasymphysis which were treated successfully by giving guiding elastics (blue color) for a period of lweek and following by MMF using wire for next 2 weeks. Mandibular fractures are often contaminated by oral bacteria. The propensity of infection is increased in the cases where the lingual mucosa is lacerated and reluctance on part of the patient to swallow or move his tongue freely so that, stasis develops with consequent accumulation of debris in the region of fracture. This causes multiplication of bacteria resulting in delayed union. In addition to this a delay in immobilization will to contamination which further leads to delayed union. Stability is considered as the best protection against infection, as movement in the presence of foreign bodies (i.e. loose screws) usually leads to infection and malunion. Infection rateis al so shown to beless with intra-oral approach. ${ }^{18}$ Avascularity is a risk factor, which should be given primary consideration and the presence of teeth in the line of fracture are others.

In the present study, patients were evaluated preoperatively and postoperatively at 2 weeks, 4 weeks, 2 months and 3 months, for the signs of infection. Pain, swelling, local rise in temperature, local inflammation and pus discharge were consi dered as indicators for the presence of infection. Out of twenty cases there was only one case of postoperative infection patient reported to our OPD with a severe through and through laceration of right side of the face exposing $\mathrm{F}-\mathrm{Z}$ region, Right Zygoma region and Left Angle region. Due to the extent of severity of the laceration it was decided to post the case in emergency and all sites were reduced. A 3D plate was used to reduce the fracture in Left Angle region and soft tissue closure was performed. Patient had numbness in the area supplied by Infra Orbital Nerve but had no Paresthesia of Mental Nerve. The infection in this case might be due to improper preparation, lack of pre-operative antibiotic infusion, improper sterilization of armamentarium or failure to maintain aseptic condition in the operation theatre. Patient was asymptomatic when discharged from the hospital, but came back with pain, swelling and opening of sutures after a week of the surgery. On examination, intraorally it was seen that there was mild wound dehiscence, pus discharge, raise in temperature, halitosis and exposure of the plate in Left Angle region. He was kept under antibiotic cover and regular oral irrigation with betadine solution. Wound closure was done using 3-0 vicryl. Patient was recalled after a week, but he did not turn up for review. Wound dehiscence was again noticed after a month. Infection did not subside which lead to the failure and subsequent removal of the implant.

With open reduction and internal fixation, the reported incidence of infection ranged from 3\% to $32 \%$. Guimond et al, reported an infection rate of $5.4 \%$ ( 2 out of 37 patients) with the use of 3D plates. ${ }^{9}$ Feledy et al, reported $9 \%$ infection rate ( 2 out of 22 patients) and Zix et al, reported $0 \%$ (0 out of 20) infection rate in their study. ${ }^{8,17}$ In the present study infection rate reported was 5\% using 3D locking plate. It had been claimed that mobility of fractured segments is a causative factor in post-operative infections. Because infection is the most common complication in mandibular fractures, the improvement of plate stability might be a way to minimize this problem. Guimond et al also experienced, low incidence of wound dehiscence and plate exposure with 3D plates in comparison to Champy's miniplate. $^{9}$

Mandible is the site associated with a relatively high incidence of altered fracturehealing (malunion, non-union). There are a number of specific risk factors associated with mandibular fractures and their potential for non-union or malunion. I nfection is the contributing risk factor to unfavorable healing and mobility. Other risk factors include poor apposition of fracture segments, presence of foreign bodies, unfavorable muscle pull on the fracture segments, displacement of comminuted fracture segments (and the difficulty associated with adequately reducing them), aseptic necrosis of bony fragments, soft tissue interposition, malnutrition and debilitation. The most common cause of failure (non-union) is, residual mobility across the fracture site. Movements of the bone ends will disrupt the 
fibro vascular structures, decrease the recruitment of osteoprogenitor cells and allow for fibrous tissue ingrowth instead of bony healing. Other contributors to fracture non-union include impaired healing capacity secondary to illness, tobacco use and infection. In some instances, there may be loss of bone, producing a continuity defect, which will require bone graft reconstruction. ${ }^{19}$

Mobility at the fractured site was examined in 20 patients preoperatively, at various follow up stages. I n our study, all patients had mobility (100\%) of fracture fragment. It was observed that 1 case (5\%) out of 20 cases had mobility after 3D Titanium locking plate fixation in $2^{\text {nd }}$ postoperative week. By the 1st postoperative month 19 (95\%) out of 20 patients showed no mobility in fractured segments, except one patient $(5 \%)$ who showed mobility at the fracturesite. At $6^{\text {th }}$ week infected plate was removed and inter dental wiring was done to reduce the mobility in that patient. At the end of $2^{\text {nd }}$ postoperative month no mobility was noticed in 19 patients. But, mild mobility was seen in 1 patient. Wiring was removed at $2^{\text {nd }}$ month when the infection was subsi ded and fracture mobility was al so absent. At the end of $3^{\text {rd }}$ postoperative month mobility was not present in any of the patients.

Rigidity of fractured segments produces a stable foundation for soft tissue growth provides improved vascularity to the area and allows better healing of wound. It also prevents bacteria from being continually pumped through the fracture site, Thereby, decreasing the chance of osteitis. It is seen that greater the mobility, greater the chance of infection. Initially, a multidisciplinary experiment was carried out by a group of engineers to check out the rigidity of monocortical plate fixation. Although this system is semi-rigid, the amount of rigidity demonstrated is sufficient for effective osteosynthesis of fractures and to resist masticatory forces during the period of healing. Recently Alper Alkan et al, carried out an in-vitro study to evaluate the biomechanical behavior of four different types of rigid fixation systems with semi-rigid fixation system that are in use currently. ${ }^{20}$ The study demonstrated that 3D struts plates had greater resistance to compression loads than the Champy's technique.

Pain associated with the procedure was recorded for all the patients based on a visual analogue scale preoperatively and post operatively at one week, two weeks, one month, two months, three months, at mandibular fracture sites. Scores for assessment of pain as follows, o- no pain, 1- Mild pain, 2- Moderate pain, and 3- Severe pain. The preoperative pain score was moderate (grade 2 ) in two patients and in 18 patients the pain was severe (grade 3).The overall average preoperative pain score was $100 \%$. On the $1^{\text {st }}$ post operative day pain was higher dueto more swelling at the operated side. At the end of 1 week pain was mild (grade 1 ) in 12 patients, moderate (grade 2 ) in 4 patients and severe (grade 3 ) in 4 patients. At the end of 2 weeks pain was decreased in 18 patients (mild), moderate in 1 patient and severe in 1 patient. At the end of 1 month pain was absent in 18 patients and one patient had moderate pain (grade 2) and another one with persisted severe pain (grade 3) which came to moderate at the end of $6^{\text {th }}$ week after removal of infected 3D Titanium locking plate. At the end of 2 months 19 patients elicited no pain and pain was moderate in one patient. At the end of $3^{\text {rd }}$ postoperative month there was no pain in all the patients.

In the present study the patients were also checked for wound dehiscence at the site of fracture, on the immediate post-operative day, after 2 weeks, 1month, 2 months, and 3 months. In our study out of 20 patients, 18 patients showed excellent postoperative healing, all of them were totally asymptomatic, and the soft tissue over the site was healthy.

In one case the sutures were opened and the wound dehiscence was seen on $1^{\text {st }}$ week postoperatively. Patient was reviewed periodically every alternative day, wound was thoroughly irrigated with saline and betadine and sutures were placed again, kept on antibiotics and continuous follow up was done, which led to satisfactory secondary healing. It was observed that by the end of 2 month healing was satisfactory. 
But in another case there was wound dehiscence observed $1^{\text {st }}$ week postoperatively and even after repeated follow ups the wound healing did not improved, infection continued to persist even after 1 month, 2 month post operatively resulting in failure of the implant by the end of 3 month.

Neurological deficit of all the 20 patients was assessed pre and postoperatively, out of 20 cases paresthesia was seen in six patients pre operatively who was diagnosed with Parasymphysis fractures, paresthesia continued postoperatively in four patients which did not subside by end of 1 month, in all these six cases intraoperatively it was found that the mental nerve was intact in four cases and the nerve was entrapped in two cases in all these cases careful dissection carried out, mental nerve was isolated and preserved, plate fixation was done. Paresthesia might be there due to swelling followed by operative procedure and retraction of the nerve during plate fixation.

Two patients elicited nerve regeneration by the end of 2 months and by the end of 3 months the remaining two patients showed complete nerve regeneration. All the cases are well appreciated to healthy functioning.

Implant Failure includes loosening of screws and breakage of the plate which was assessed in all the 20 patients post operatively both clinically and radiographically on $1^{\text {st }}$ postoperative day, at 2 weeks, 4weeks, 2 month and 3 month interval. Among the 20 patients, 19 patients showed no implant failure, whereas in one case loosening of the screws was documented. Farmand $\mathrm{M}$ reported a good stability against traction forces and torsional forces with 3D plating system. ${ }^{6}$ Witten berg et al also reported that 3D plating system may provide adequate fixation for mandibular fractures. ${ }^{8}$ In light of the evidence provided by the literature, in our study all the cases fracture fragments were found to be stable after platefixation and no inter fragmentary mobility was observed, thus ascertaining that 3D plate was superior in respect of stability.

\section{Conclusion:}

The present study concludes that 3D plates stabilize the bone fragments in three dimensions because of closed quadrangular geometric shape, and the ease of contouring and adapting. Due to better interfragmentary stability, supplemental fixation in the form of IMF/MMF is not necessary, thereby enhancing the overall comfort, convenience and well being of the patients. 3D plate holds the fracture segments rigidly by resisting the 3D forces namely shearing, bending and torsional forces occurring on thefracture site in function. Our clinical results and biomechanical investigations have shown a good stability of the 3D plates in the osteosynthesis of mandibular fractures without major complications. The thin connecting arms of the plate allow easy adaptation to the bone without distortion. The free areas between the arms permit good blood supply to the bone. The 3D plate allows no movement at the superior and inferior borders with maximal torsional and bending forces as opposed to a single linear plate applied to superior border area. When onelinear plate is placed at the superior border area, torsional and bending forces usually cause the movement along the axis of the plate with buccolingual splaying and gap formation at the inferior borders, respectively. All the patients in our study appreciated early recovery of normal jaw function, uneventful healing and good union at the fracture site with minimal weight loss due to early reinstatement of the masticatory function. There was great patient acceptance of this treatment modality. 3D plates were indeed easy and simple to use. Significant reduction in operating time could be achieved with the use of 3D plates which makes it a time-saving alternative to conventional miniplates. Placement of 3D plate was found to be more comfortable to the surgeon. The 3D plate was found to be standard in profile, strong yet malleable, facilitating reduction and stabilization at both the superior and inferior borders giving threedimensional stability at fracture site. The small sample size and short duration of follow up could be considered as the limitations of our study. It is hence recommended to have a multicenter study with large number of patients and correlation among these studies to authenticate our claims. 


\section{References}

1. Raymond J Fonseca. Oral and Maxillofacial Trauma. Pennsylvania, WB Saunders Company, $2^{\text {nd }}$ Edition, Vol 1, 1991.

2. Pavan Kumar B, J eevan Kumar, Mohan AP, Venkatesh V, and Rahul. A comparative study of three dimensional stainless steel miniplates in the management of mandibular Parasymphysis fracture. J Bio Innov 2012; 1(2): 19-32.

3. Booth Peter Ward. Trauma: Surgical management of mandibular fractures. In: Booth Peter Ward, Schendel Stephen A, Hausamen J arg-Erich (eds), $2^{\text {nd }}$ edition Maxillofacial Surgery, vol. 1. Edinburgh: Churchill Livingstone 2007, 74-76.

4. Sauerbier S, Schon R, Otten J E, Schmelzeisen R, Gutwald $\mathrm{R}$. The devel opment of plate osteosynthesis for the treatment of fractures of the mandibular body - A literature review. J Craniomaxillofac Surg 2008; 36:251-9.

5. Gear AJ , Apasova E, Schmitz J P, Schubert W. Treatment modalities for mandibular Anglefractures.J Oral Maxillofac Surg 2005; 63:655-63.

6. Farmand M, Dupoirieux L. The value of 3-dimensional plates in maxillofacial Surgery. Rev Stomatol Chir Maxillofac 1992; 93:353-7.

7. Manoj Kumar J ain, K Sankar, C Ramesh, Ramakrishna Bhatta. Management of mandibular interforaminal fractures using 3 dimensional lockingand standard titanium miniplates e A comparative preliminary report of 10 cases. J Cranio Maxillo Facial Surgery 2012; 40:475-8.

8. Feledy J, Caterson Edward J, Shon S, Samuel S, Larry H, Lee C. Treatment of mandibular angle fractures with a matrix miniplate: A preliminary report. Plastic Reconstructive Surgery 2004; 114:1711-8.

9. Guimond C, J ohnson J V, Marchena J M. Fixation of mandibular angle fractures with a 2.0- $\mathrm{mm}$ 3- dimensional curved angle strut plate. J Oral Maxill ofac Surg 2005; 63:209-214.
10. J ain MK, Manjunath KS, Bhagwan BK, Shah DK: Comparison of 3-dimensional and standard miniplate fixation in the management of mandibular fractures. J Oral Maxillofac Surg 2010; 68:1568-72.

11. Mohit Agarwal, Balram Meena, D.K. Gupta, Anjali Dave Tiwari, and Sunil Kumar J akhar. A prospectiverandomized clinical trial comparing 3D and standard miniplates in treatment of mandibular Symphysis and Parasymphysis fractures. DOI 10.1007/s12663-013-0483-x.

12. Vijay Ebenezer, Balakrishnan Ramalingam. ThreeDimensional Miniplate Fixation in Mandibular Angle Fractures. Ind J Multidisciplinary Dent 2011; 1(2).

13. I izuka T, Fujimoto H, OnoT. A new material (single crystal sapphire screw) for internal fixation of the mandibular ramus. J Craniomaxillofac Surg 1987; 15(1):24-7.

14. Michelet FX, Deymes J, Dessus B. Osteosynthesis with miniaturized screwed plates in maxillofacial Surgery. J ournal Maxillofac Surg 1973; 1:79.

15. Champy M, Lodde J P, Schmitt R et al. Mandibular osteosynthesis by miniature screwed plates via a buccal approach. J ournal Maxillofac Surg 1978; 6:14-21.

16. Gaurav Mittal, Ramakanth Reddy Dubbudu. Three dimensional titanium mini plate In Oral and Maxillofacial Surgery. A Prospective Clinical Trial. J Maxillofac Oral Surg 2012; 11(2):152-9.

17. Zix J , Lieger O, lizuka T. Use of straight and curved 3 dimensional titanium miniplates for fracture fixation of the mandibular angle. J Oral Maxillofac Surg 2007; 65:1758-63.

18. Zachariades N, Papademetriou I, Rallis G. Complications associated with Rigid Internal fixation of facial bone fractures. J Oral Maxillofac Surg 1993; 51:275-8.

19. Bochlogyros PN. A retrospective study of 1521 mandibular fractures. J Oral Maxillofac Surg 1985; 43:597

20. Alkan A, Celebi N, Ozden B, Bas B, I nal S. Biomechanical comparison of different plating techniques in repair of mandibular angle fractures. Oral Surg Oral Med Oral Pathol Oral Radiol Endod 2007; 104:752-6.

\section{Gain quick access to our journal online View our journal at www.nacd.in}

